\title{
Application of MRI for improved local control in complex radiotherapy of cervical cancer
}

Janaki Hadjiev, Zsolt Cselik, Péter Bogner, Árpád Kovács, Ferenc Lakosi, Gyula Kotek, Imre Repa

\begin{abstract}
Background: The aim of this study was to analyze the use of magnetic resonance imaging (MRI) as a modern medical imaging technique in radiotherapy with special emphasis on the integration of MRI and a novel technique in brachytherapy to optimize treatment outcome in cervical cancer.

Methods: In addition to the CT based shrinking volume conformal teletherapy in 31 patients with locally advanced cervical cancer, MRI examination with a special adjustable applicator at the treatment site was performed for the brachytherapy planning. To avoid excessive doses to the healthy structures during complex cervical radiotherapy isodose curves were calculated upon the information of the MR image and dose distribution was evaluated.

Results: The consecutive application of CT and MRI limited the possibility for overdosage of the critical organs and undertreatment of the advanced tumor spread in all cases. The overall response rate for the complex treatment was $74.2 \%$ with complete regression in $25.8 \%$ of the cases. Based on the exact information of the three dimensional digital data radiation doses could be optimized without increasing the possibility of acute complications rate.

Conclusion: The introduction of $3 D$ treatment planning for teletherapy pelvic and boost irradiation of cervical carcinoma as well as for the brachytherapy part of the complex treatment is to be recommended.
\end{abstract}

KEY WORDS: Uterine Cervical Neoplasms; Magnetic Resonance Imaging; Brachytherapy; Radiotherapy Dosage; Radiology, Interventional

University of Kaposvár, Health Sciences Center, Kaposvár, Hungary; Address correspondence to: Janaki Hadjiev, Health Sciences Center, University of Kaposvár, Guba Sándor u. 40, H-7400 Kaposvár, Hungary; E-mail: Janaki.Hadzsiev@sic.hu; The manuscript was received: 01.08.2006, Provisionally accepted: 05.10.2006, Accepted for publication: 05.10 .2006

(c) 2006, Institute of Oncology Sremska Kamenica, Serbia

\section{INTRODUCTION}

Exacts xact staging of cervical carcinoma is essential in selecting the most favorable therapy. ing system there are significant inaccuracies. With a 24\%-39\% error rate in gynecologic examinations also the degree of the pelvic invasion is often inadequately evaluated without cross-sectional diagnostic imaging (1-4). Furthermore, lesion volumes, nodal, and distant metastases are not assessed. The broad scale of imaging modalities evolved during the last two decades contributes to a better knowledge and management of the disease. Magnetic resonance imaging (MRI) is now widely accepted as optimal for evaluation of the main prognostic factors and selection of therapeutic strategy (5-7).

In advanced disease or when bad prognostic factors are present, the complex oncological treatment is based on the combination of external irradiation and brachytherapy with concomitant chemotherapy. Nevertheless, optimal protocol for the determination of the proper therapeutic plan remains to be defined. An unified technique to solve the problems connected to precise definition of the gross tumor volume (GTV) and the clinical target volume (CTV) with their geometrical change during the external irradiation as well as their dose covering during conformal HDR brachytherapy is nowadays becoming widely available (8). Reproducibility of the treatment plan along with a stable multiple exact repositioning of the patient and the treatment device is essential for the clinical outcome of the treatment.

\section{PATIENTS AND METHODS}

\section{Patient selection}

Between April 2002 and February 2004, 31 patients with locally advanced uterine cervical cancer (International Federation of Gynecology and Obstetrics (FIGO) stage distribution: IIb-IVa) completed combined external beam radiotherapy (EBRT) and brachytherapy exclusively or in conjunction with concomitant chemotherapy.

\section{Radiotherapy}

For the period of the prospective study a standard radiotherapy treatment protocol was applied containing CT based shrinking volume conformal EBRT, and MRI-assisted brachytherapy with a special adjustable applicator. Three-dimensional conformal radiation treatment planning and delivery was used for the radiotherapy with the general purpose of shaping the prescribed dose volume to the form of the 3-dimensional target volume, simultaneously limiting dose to critical normal structures. Initially a CT-image based, planning target volume (PTV) was treated with a four-field box technique of EBRT on a Mevatron Primus 15 MV linear accelerator (Siemens, Erlangan, Germany). After EBRT with a median dose of 50.6 (range 48.8 - 54.2) Gy in 26 (range: 25-28) fractions over 4-5 weeks to the PTV, the radiation dose was boosted to 61.4 (range 59.8 - 65) Gy.

After the completion of the boost EBRT, a flexible $5 \mathrm{~F}(1.65 \mathrm{~mm})$ interstitial cervical applicator (Cook ${ }^{\circledR}$ BFCS-6.OR-30-STB-25, Bloomington, USA) insertion and fixation were performed. The technique and its benefits for the patient and the radiation oncologist had been described in details in a previous study (9). 


\section{MRI}

High-resolution T2-weighted fast-spin-echo (TE 95.0, TR 4800.0, FOV 28) MR images (5-mm section thickness) (512x512 matrix) were obtained in sagittal and axial planes from the promontorium to the vulva with the applicator and the patient in the treatment position (Figure 1).
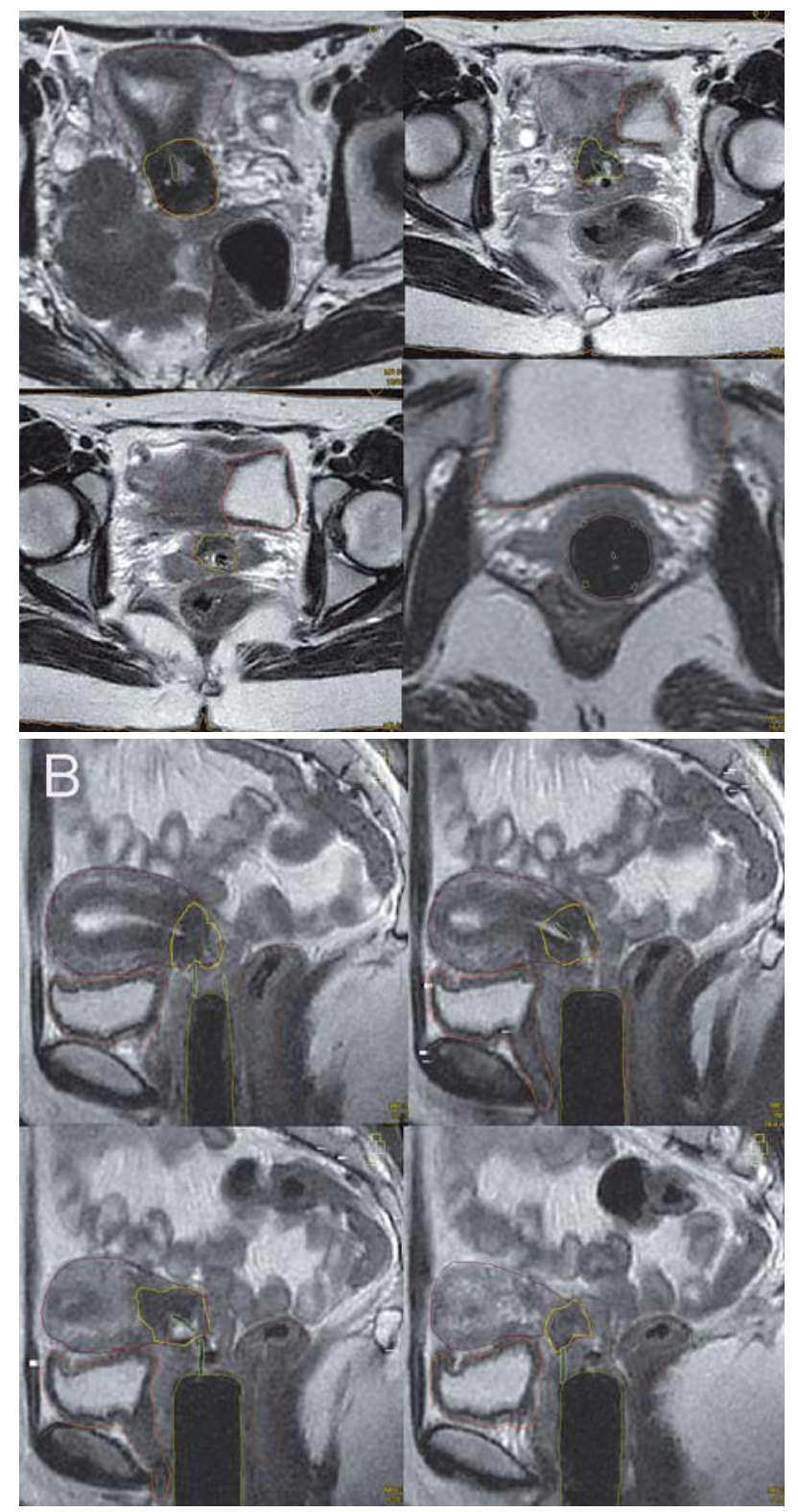

Figure 1. (A) T2 weighted MR images in axial plane from different levels, with the patient and the applicator device in treatment position. The applicator geometry is accurately visualized as well as the surrounding tissue and the cervical cancer extension. (B) T2 weighted MR images in sagittal plane, with the patient and the applicator device in treatment position. CTV and the organs at risk are delineated on all planes

Decreased imaging time and increased image resolution proved to be an advantage of the body coil in combination with fast spin-echo T2-weighted imaging technique, without loss of staging accuracy.

In cases of pelvic soft-tissue edema a fat-saturated T2-weighted sequence was used. This sequence often did not demonstrate sufficient contrast between the lesion and the intact part of the gynecologic organs and the visualization of the applicator was difficult. Still in the cases mentioned above, it proved to be the most-suitable for the differentiation of the tumor spread to the surrounding tissue.
When a histologically verified cervical lesion was not clearly detectable on the T2-weighted images additional $6 \mathrm{~mm}$ slice thickness dynamic gadolinium-enhanced, fat suppression T1 imaging (TE 11.0, TR 734.0, FOV 200) was used for the evaluation and contouring (Figure 2). Complete disruption of the stromal ring with nodular or irregular signal intensity extending into the parametrium was taken for a still present invasion.

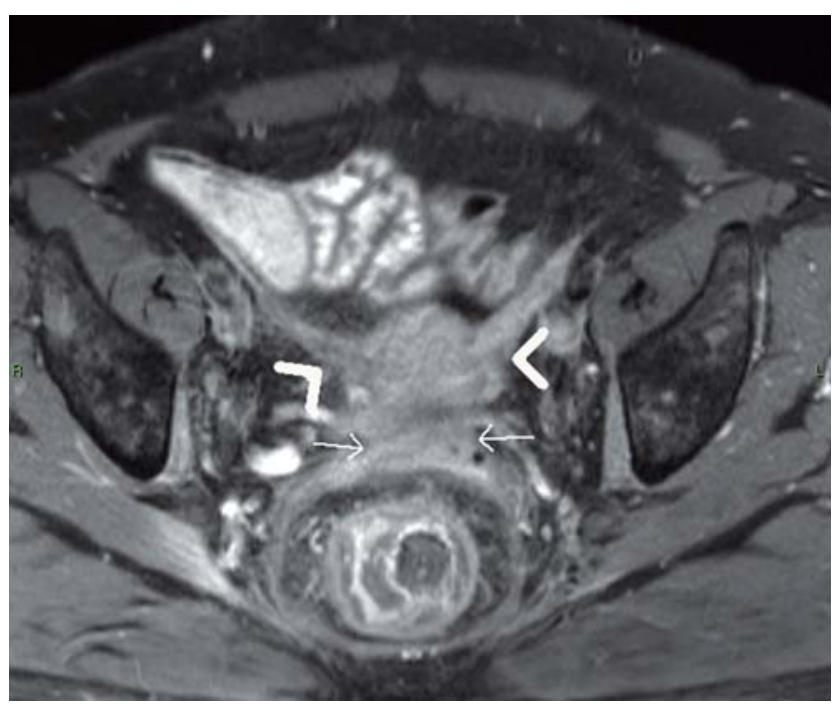

Figure 2. Gadolinium-enhanced fat-saturated T1-weighted axial cross-section. Spread of tumor tissue to the vagina (arrows) and parametrium is well depicted (arrow heads)

During the delineation of the CTV a contour disruption of the vaginal wall with hyperintense thickening on the T2-weighted images, or contrast material enhancement on the T1weighted imaging were considered as signs of vaginal invasion.

Clinical findings for the exact estimation of the vaginal extension were compared to the MRI findings and also taken in consideration.

\section{Treatment planning}

Sequential MR images were used in delineation of the CTV for the BT and the organs at risk (OARs) on all planes. The contour was individually tailored taking into concern the MRIdefined GTV, where the density of the cancer cells is the highest and the initial, pretreatment tumor extension (Figure 1).

Three dimensional reconstruction and rotation of the structures delineated on the MR images facilitated better visualization of the volumes as well as the relationship between the organs and the applicator device (Figure 3).

A conformal BT plan was calculated with the Abacus ${ }^{\mathrm{TM}}$ dose-planning system (MDS Nordion ${ }^{\circledR}$, Quebec, Canada). The generated isodose lines and CTV contours were superimposed for each axial MR image and were also reconstructed in the sagittal and coronal views to visualize the 3D coverage (Figure 4). Dose adaptation to the CTV was based on the volumetric measurements for the GTV and the critical organs. Dose volume histograms were calculated to evaluate the dose that covers $100 \%$ and $90 \%$ of the target volume and doses to specific absolute volumes of organs at risk. The limits defined for the OARs (rectum and bladder) were 4 Gy per fraction for tissue volume of 2 and $4 \mathrm{~cm}^{3}$, respectively.

\section{Treatment}

With the application of the MRI determination of the rectal reference point was done slightly differently from the recommendation of International Commission on Radiation Units and Measurement (ICRU) Report 38 (10). The possible need for a revision of the report written in 1985, due to the dramatic progress in imaging, planning and therapy equipment avaliability, was already raised by Pötter and al in 2001(11). The maximum rectal dose was calculated at the points in the anterior wall of the rectum closest to the applicator and receiving the maximum dose. 


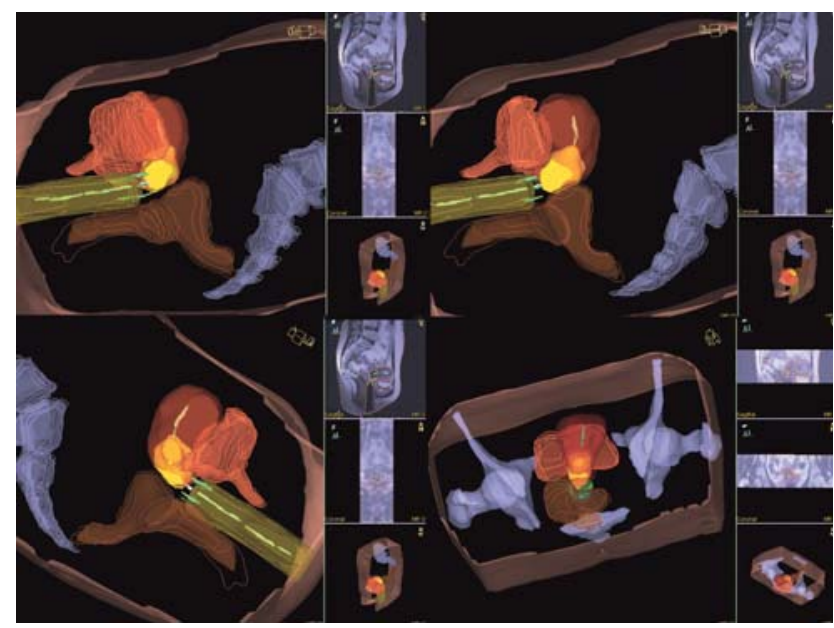

Figure 3. 3D reconstruction of MR images. The cylindrical holder (transparent dark green) with the central and circumferential applicators (light green), the CTV (yellow), the uterus and the OARs; the rectum and the bladder (dark-red, brown and orange respectively), and the bone structures (gray) are presented. Rotation facilitates better visualization of the volumes as well as the relationship between the structures and the applicator device

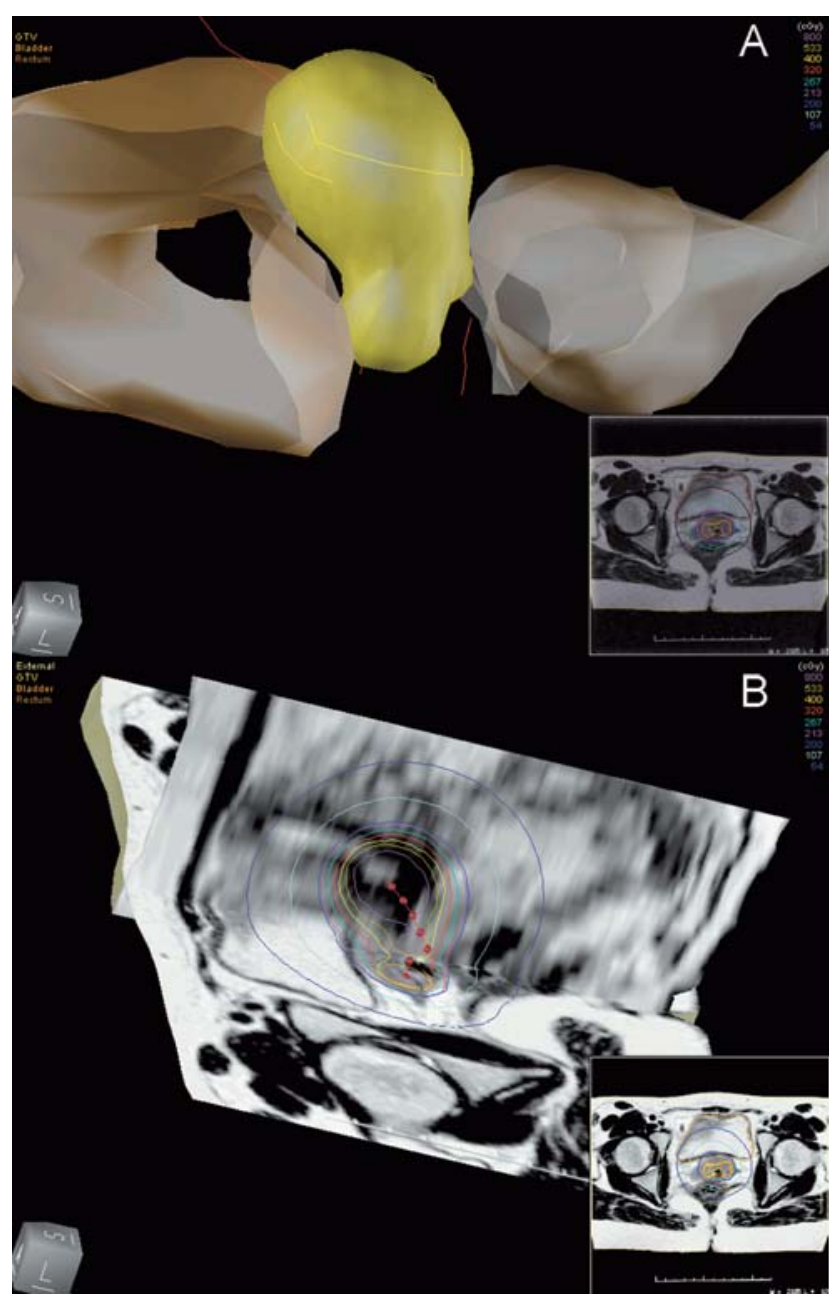

Figure 4. MR-assisted 3D treatment plan. (A) Three dimensional volumetric representation the GTV/CTV (lines), the OARs (transparent surface) and the 100\% (4Gy) isodose coverage (yellow solid surface). (B) The CTV and the OARs are presented with the isodose coverage on a sagittally and axially opened $3 \mathrm{D}$ reconstruction
Change of cervix and upper vagina anatomy during the course of the treatment reported in the literature was not taken in consideration for the HDR ICB as all the treatments were individually planned and performed in a short time interval after the greater volumetric change caused by the EBRT $(12,13)$.

The high-dose-rate (HDR) intracavital BT was carried out with a GammaMedplus ${ }^{\mathrm{TM}}$ remote after-loading machine (Varian Medical Systems Inc. Palo Alto, USA), with ${ }^{192}$ Ir stepping sources. With the aim to improve the local control rate with fewer complications the brachytherapy was performed in three fractions, twice a week, with a total dose of $12 \mathrm{~Gy}$ prescribed to $\geq 90 \%$ of the CTV.

The overall duration of treatment ranged from 50 to 62 days (median, 54 days).

\section{RESULTS}

The application of MRI limited the possibility for overdosage of the critical organs and undertreatment of the advanced tumor spread in all cases. The spatial relationship of the tumor to the bladder, rectum, bowel, applicators, etc. was depicted well. Doses to the tumor and surrounding normal tissues were read from isodose curves superimposed on the T2weighted sagittal image, which was useful in customizing the dose distribution (Figure 5).

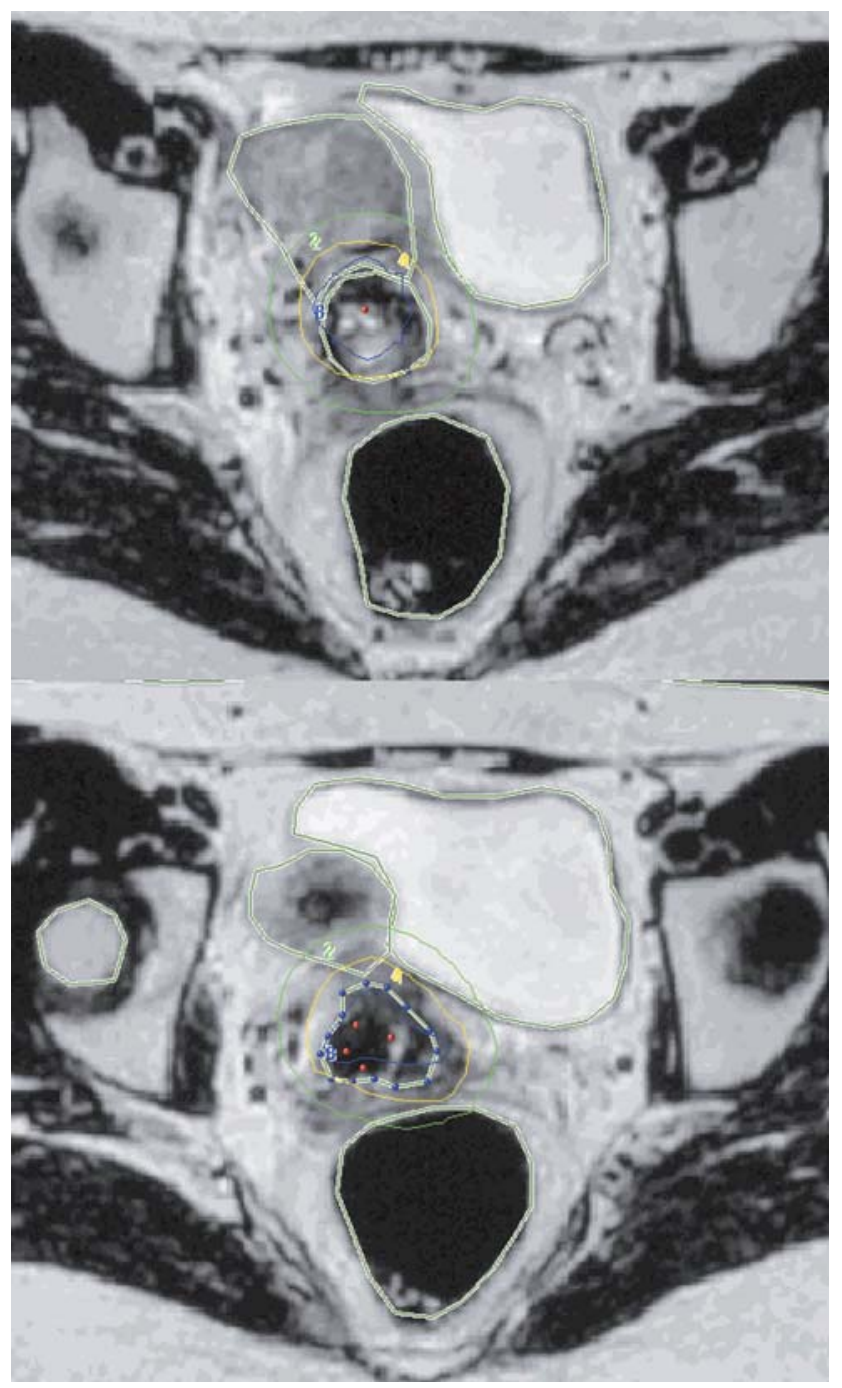

Figure 5. MRI-based conformal HDR BT plan. T2 weighted axial MR image with the applicator in treatment position and the prescribed fraction isodose curves for 2, 4 and 8 Gy. The delineations of the CTV (blue dots) and the OARs; the rectum and the bladder on the sectional images are imported directly into the treatment planning system, and the dose-volume association is evaluated. 
In this manner a reduction of the treated volume could be achieved leading to a possible further decrease of complications. In majority of the treatments, rectum and bladder doses were less than $70 \%$ of target dose due to the rapid fall-off of the brachytherapy dose and the conformal visualization provided by the MRI. However on very few treatment plans, in order to obtain sufficient covering of the CTV the bladder dose was higher than the required. In those cases, the vaginal holder of the applicator device was reseated for better tolerance of late responding normal tissues. Dose calculations were checked with an independent calculation method and agreement was obtained within 5-7\% discrepancy.

As primary end points, the coverage of the PTVs and the CTV, the dose to the OARs, the acute toxicity and CT examination was performed for the local tumor control evaluation. Acute adverse events were graded on the basis of Common Terminology Criteria of Adverse Events Version 3.0 (CTCAE). The overall response was determined by the Response Evaluation Criteria in Solid Tumors (RECIST) guidelines (14).

The treatment proved feasible and was tolerated well by all patients. There was no treatment related death. Grade 1 adverse events were evaluated with some difficulties because of the minor degree of symptoms, as well as because of the fact that they mostly depended on subjective perception. As adverse events evaluation forms were filled in by the patients before initiation of the treatment some of the events proved to be preexisting. In those cases only the rise of complaints was taken in consideration. Grade 1 diarrhea (with occasional abdominal pain) occurred in 6 patients (36\%) during the $4^{\text {th }}$ week of the EBRT. Following a decrease of the daily dose and a strict adherence to the proposed diet, the clinical signs had disappeared by 1 week after the completion of the irradiation. The occurrence of bladder complications was less frequent (3 patients, 18\%), but more severe (Grade 2; acute sterile cystitis with bladder spasm), in the last week of the EBRT. Antibiotics, antispasmolytic treatment and an extensive daily fluid intake led to normalization of the patients' condition. Insertional or acute complications related to the BT were not observed.

In acceptance with the RECIST guidelines no non-target lesions were found during the baseline documentation and no novel tumorous lesion appeared during the course of the treatment. From this respect the overall tumor response to the complex oncological treatment was identical with the response of the target lesion. Applying the linear-quadratic model for sublethal damage repair (tumor $\alpha / \beta=10,0 A R \alpha / \beta=3$ ) the dose of the brachyterapy treatment was biologically normalized to the EBRT dose fractions. The PTV, PTV-boost and CTV median coverage was $97.4 \%, 98.8 \%$ and $93.2 \%$ respectively. Thus, the prescribed total dose, calculated from the parameters of the two irradiation modalities was received by $17.7 \%$ and $13.3 \%$ of the total volume of the OARs' (the rectum and the bladder respectively). Both the coverage of the PTVs and the CTV and the radiation burden on the OARs were within acceptable limits.

The postreatment CT examination and the gynecological physical examination used as evaluations for local tumor control showed overall response rate for the complex treatment as $74.2 \%$. Clinical results were; complete regression in $8(25.8 \%)$, and partial regression in other 15 patients (48.4\%). In 7 cases (22.6\%), a moderate treatment response was achieved, where the disease was considered stable, and poor in 1 patient (3.2\%), who displayed progression of the disease.

\section{DISCUSSION}

Tumor stage, tumor size and nodal status are the most important prognostic factors in cervical cancer. Anteroposterior tumor diameter predominantly affected the incidence of distant metastasis, and lymph node status affected both pelvic control and distant metastasis. Since 1984 when Bies and his coworkers published their experience in comparison of clinical staging with the CT, MR and surgical findings in gynecological tumors magnetic resonance imaging plays a more and more important role in the accurate staging of cervical malignancies (15).

With a prospective study it was shown that endosonography and MRI are more accurate than CT in the local staging (16). MRI-based diagnosis enables the determination of a correct tumor staging preoperatively, and is therefore very helpful in planning an adequate therapy. With MRI excellent imaging of the tumor spread within the cervix can be achieved, as well as also tumor extension to the parametrium and infiltration of the neighboring structures (17-19). To date MRI is the method of choice in the preoperative staging of cervical cancer and if used more widely it would contribute to simplification and shortening of the preoperative diagnostic procedure in patients with cervical carcinoma (19).

Over the last 15 years, treatment of cervical cancer has become increasingly sophisticated with advances in external beam and brachytherapy in the radiotherapeutic management of this carcinoma.

Treatment for cancer of the uterine cervix, prior to 1998 originally planned upon radiographic evaluation proved to be insufficient in the knowledge of to what extent the treated volume encompasses the CTV and studies came into view with CT-based treatment planning $(20,21)$. The radiography based planning provides only dimensions and doses at selected points, which are difficult to be uniformly defined and exactly reproduced. Even when used in combination with physical pelvic examination this technique in spite of its cost-effectiveness and convenience, seems nowadays not acceptable.

Beside the reported high percentage accuracy of MR imaging in staging of cervical carcinoma the modality has a great importance in the treatment of cervical cancer (22). Regarding therapy the role of MRI may be classified in three main groups; 1) guided applicator positioning; 2) assisted treatment planning and; 3) treatment quality control.

MR imaging provides three-dimensional (3D) anatomic information with capability of three-dimensional measurement and has great advantages in terms of soft-tissue contrast resolution. The subdivision of different approaches and the transfer from point doses to volumes in treatment planning is possible and practical for the treatment of cervix carcinoma in brachytherapy. At the same time MRI is the only imaging modality giving the opportunity for an accurate judgment of the invasion towards the surrounding normal tissue. Thus MRI based planning provides proper information on target and organ volumes and dose-volume histograms.

Since Schoeppel published in 1992 his experience in three patients with MRI during gynecological brachytherapy treatment the need for integration of the two techniques became essential (23). Data for conformal brachytherapy dose prescription and treatment planning found in the literature still lag behind the state-of-the-art for EBRT. Tardivon and his group found MR imaging useful in controlling the relationships between the tumor and the applicator as well as it facilitated treatment planning, since the radiation dose to the tumor volume and adjacent critical organs could be calculated accurately with no false-negative results observed (24). The comparative use of the MRI for the 3D brachytherapy planning system, with the applicators on site, allows dose escalation in the GTV required for the effective radiation treatment with maximal protection of the surrounding healthy tissues.

Together with the use of MRI assisted planning for brachytherapy a crucial need for an improvement in the applicator technique appeared. The following conditions and terms had to be available: 1) the applicator had to be MR-compatible and visible on MRI, CT and X-ray screen and film; 2) thin diameter was crucial as the applicator had to be suitable for insertion through the cervical channel narrowed by the tumor mass; 3) adequate dosage was needed in the cases, when tumor extension involves the mid and lower vaginal mucosa; 4) during the gauze packing of the ovoids, either operator error or narrowing of the vaginal apex could result in mal-alignment of the colpostats and subsequent inadequate dosing to the exo-cervix.

The vaginal cylinder used in our study has been designed to address these concerns being equipped with the circumferential holes.

The presented technique gives also an opportunity for a MRI guided conversion of intracavitary BT to interstitial BT by means of a simple change of the applicators located in circumferential channels of the adjustable vaginal cylinder holder to MR-compatible needles (Figure 6). This may be of appreciable importance in patients with disease that cannot be optimally encompassed by intracavitary BT in order to achieve more accurate volume optimization of dose distribution. 


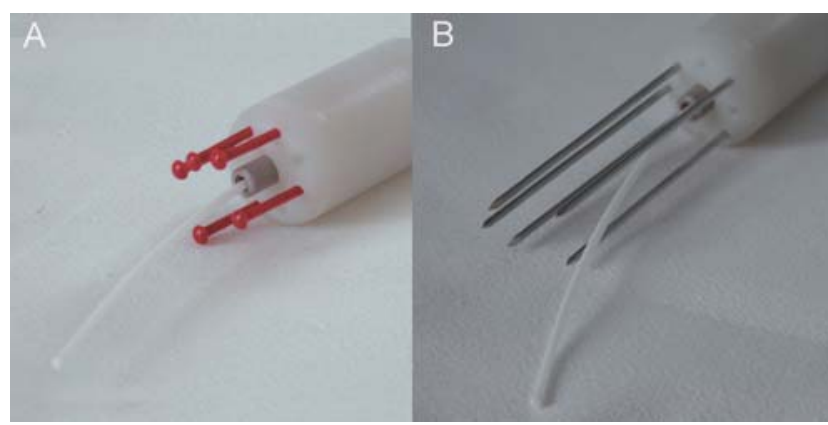

Figure 6. (A) The intracervical applicator introduced through the central channel of the cylindrical holder used for vaginal interposition. (B) The applicator device with MR compatible needles in the circumferential channels

There is a belief that it is unnecessary to calculate doses for each HDR insertion beyond the first one. This conviction is based mainly on the fixed geometry applicator. It has been shown even though the applicator may have reproducible geometry, that it is difficult to insert the applicator reproducibly from one insertion to another (25). It is generally supposed that all insertions are geometrically identical to the first, so dosimetric errors at one or more of the dose points for subsequent insertions may be significant. This dose specification method results in underdosing of important target tissues or overdosing of adjacent dose-limiting structures (26). On the other hand the ovoid sources of the frequently used applicators do not always contribute to local control, and occasionally lead to rectal complications (27). Thus optimization of brachytherapy depends on patients' anatomy, tumor size, and tumor response. The single insertion, the fixation to the cervix and the MRI assistance allows a proper volumetric dose-planning lowering the possibility for those dosimetric errors. Evaluation of dosevolume histograms of critical organs and tumor within the reference isodose volumes could be performed using MRI assisted three-dimensional planning and computed dosimetry. The rectal and bladder reference points may not represent the points of maximum dose delivery to these organs. Without the use of cross-sectional image assistance, despite the determination of multiple reference points this information is inadequate to predict doses to the entire rectum and bladder, since single point measurements at the bladder neck seriously underestimate the dose to the bladder. Dose-volume histogram already established as a gold-standard in the EBRT is giving a precise dose evaluation of the OARs. Together with the better interpretation of target delineation, delineation of critical structures as well as dose distribution conformal brachytherapy treatment planning for interstitial brachytherapy means significant advantage for the clinical routine compared to $2 \mathrm{D}$ or semi-3D methods. Cross-sectional image assisted brachytherapy may be inadequately criticized for being a time consuming process with high costs. In those institutes, where the MRI based treatment planning has been systematically introduced into daily clinical practice and is routinely used, planning procedures on both $2 \mathrm{D}$ (semi-3D) and 3D planning systems have a similar time consumption $(28,29)$.

\section{CONCLUSION}

Fractionated high dose rate brachytherapy can be given with both higher dosimetric accuracy and more adequate irradiation of the tumor component, if it is MRl-assisted and with a single applicator insertion. With this new applicator, it was possible to accumulate very accurate and detailed 3D dose-distribution data for the critical structures and other points of interest in the vicinity of the applicator. These data makes possible the not only the practical transfer from point doses to volumes in treatment planning, but will permit also future analysis of the correlation of dose and outcome for carcinoma of the cervix.

The increase of the therapeutic window by the systematic integration of the MRI into treatment planning, allows it to be highly individualized and with the use of MR-compatible, adjustable applicator allows safe and reproducible cervical radiotherapy with no added discomfort or hazard for the patient.

\section{REFERENCES}

1. McCarthy $S$. The uterus and vagina. In: Higgins $C B$, Hricak $H$, Helms $C A$, editors. Magnetic resonance imaging of the body. 3rd ed. New York: Lippincott-Raven; 1974. p.761-814.

2. Reinhold C. Uterus and cervix. In: Semelka RC, Ascher SM, Reinhold C, editors. MRI of the abdomen and pelvis: a text atlas. New York: Wiley-Liss; 1997. p.585-660.

3. Subak LL, Hricak H, Powell B, Azizi L, Stern JL. Cervical carcinoma: computed tomography and magnetic resonance imaging for preoperative staging. Obstet Gynecol 1995;86:43-50.

4. Togashi K, Morikawa K, Kataoka ML, Konishi J. Cervical cancer. J Magn Reson Imaging 1998;8:391-7.

5. Choi SH, Kim SH, Choi HJ, Park BK, Lee HJ. Preoperative magnetic resonance imaging staging of uterine cervical carcinoma: results of prospective study. J Comput Assist Tomogr 2004;28(5):620-7.

6. Okamoto $\mathrm{Y}$, Tanaka YO, Nishida M, Tsunoda H, Yoshikawa H, Itai Y. MR imaging of the uterine cervix: imaging-pathologic correlation. Radiographics 2003;23(2):425-45.

7. Özsarlak Ö, Tjalma W, Schepens E, Corthouts B, Op de Beck B, Van Marck E, et al. The correlation of preoperative $\mathrm{CT}$, MR imaging, and clinical staging (FIGO) with histopathology findings in primary cervical carcinoma. Eur Radiol 2003;13(10):2338-45.

8. Pötter R, Haie-Meder C, Van Limbergen E, Barillot I, De Brabandere M, Dimopoulos J, et al. Recommendations from gynaecological (GYN) GEC ESTRO working group (II): Concepts and terms in $3 \mathrm{D}$ image-based treatment planning in cervix cancer brachytherapy-3D dose volume parameters and aspects of $3 \mathrm{D}$ image-based anatomy, radiation physics, radiobiology. Radiother Oncol 2006;78(1):67-77.

9. Hadjiev J, Antal G, Antalffy Z, Bogner P, Esik O, Repa I. A novel technique with a flexible applicator fpr MRI-based brachytherapy of cervical cancer. Eur J Gynaecol Oncol 2004;3:347-50.

10. International Commission on Radiation Units and Measurements (ICRU, 1985), Dose and Volume Speciification for Reporting Intracavitary Therapy in Gynecology, ICRU Report, 38. Bethesda, MD; ICRU.

11. Pötter R, Van Limbergen E, Gerstner N, Wambersie A. Survey of the use of the ICRU 38 in recording and reporting cervical cancer brachytherapy. Radiother Oncol 2001;58(1):11-8.

12. Elhanafy $O A$, Das RK, Paliwal $B R$, Migahed MD, Sakr HA, Elleithy M. Anatomic variation of prescription points and treatment volume with fractionated high-dose rate gynecological brachytherapy. J Appl Clin Med Phys 2002; (1):1-5.

13. Dimopoulos J, Lang S, Kristis C, Böswarth J, Bauer N, Wachter-Gerstner N, et al. Combined MRI-based intracavitary and intersitial brachytherapy for locally advanced cervical cancer with a modified tandem ring applicator-clinical feasibility and preliminary results. Radiother Oncol 2004;71:S10.

14. Therasse P, Arbuck SG, Eisenhauer EA, Wanders J, Kaplan RS, Rubinstein L, et al. New guidelines to evaluate the response to treatment in solid tumors. European Organization for Research and Treatment of Cancer, National Cancer Institute of the United States, National Cancer Institute of Canada. J Nat Cancer Inst 2000;92(3):205-16.

15. Bies JR, Ellis JH, Kopecky KK, Sutton JP, Klatte EC, Stehman FB, et al. Assessment of primary gynecologic malignancies: comparison of $0.15-T$ resistive MRI with CT. AJR Am J Roentgenol 1984;143(6):1249-57.

16. Cobby M, Browning J, Jones A, Whipp E, Goddard P. Magnetic resonance imaging, computed tomography and endosonography in the local staging of carcinoma of the cervix. Br J Radiol 1990;63(753):673-9.

17. Michniewicz $\mathrm{K}$, Oellinger J. Diagnostic imaging in invasive cervical carcinoma: MRI, CT, and ultrasonography. Zentralbl Gynakol 2001;123(4):222-8.

18. Oellinger JJ, Blohmer JU, Michniewicz K, Siewert C, Wust P, Gutberlet M, et al. Pre-operative staging of cervical cancer: comparison of magnetic resonance imaging (MRI) and computed tomography (CT) with histologic results. Zentralbl Gynekol 2000;122(2):82-91.

19. Pecorelli S, Odicino F. Cervical cancer staging. Cancer J 2003;9(5):390-4.

20. Fellner C, Pötter R, Knocke T, Wambersie A. Comparison of radiography and computed tomography-based treatment planning in cervix cancer in brachytherapy with specific attention to some quality assurance aspects. Radiother Oncol 2001;58(1):53-62. 
Hadjiev J. et al.

21. Haie-Meder C, Potter R, Van Limbergen E, Briot E, De Brabandere M, Dimopoulos J, et al. Gynaecological (GYN) GEC-ESTRO Working Group.Recommendations from Gynaecological (GYN) GEC-ESTRO Working Group (I): concepts and terms in 3D image based 3D treatment planning in cervix cancer brachytherapy with emphasis on MRI assessment of GTV and CTV. Radiother Oncol 2005;74(3):235-45.

22. Nicolet V, Carignan L, Bourdon F, Prosmanne O. MR imaging of cervical carcinoma: a practical staging approach. Radiographics 2000;20(1):1539-49.

23. Schoeppel SL, Ellis JH, LaVigne ML, Schea RA, Roberts JA. Magnetic resonance imaging during intracavitary gynecologic brachytherapy. Int J Radiat Oncol Biol Phys 1992;23(1):169-74.

24. Tardivon AA, Kinkel $K$, Lartigau E, Masselot J, Gerbaulet AP, Vanel D. MR imaging during intracavitary brachytherapy of vaginal and cervical cancer: preliminary results. Radiographics 1996;16(6):1363-70.

25. Datta NR, Basu R, Das KJ, Rajasekar D, Pandey CM, Ayyagari S. Problems in reporting doses and volumes during multiple high-dose-rate intracavitary brachytherapy for carcinoma cervix as per ICRU Report 38: a comparative study using flexible and rigid applicators. Gynecol Oncol 2003;2:285-92.

26. Mai J, Erickson B, Rownd J, Gillin M. Comparison of four different dose specification methods for high-dose-rate intracavitary radiation for treatment of cervical cancer. Int J Radiat Oncol Biol Phys 2001;51(4):1131-41.

27. Ohizumi Y, Akiba T, Imamiya S, Tamai Y, Mori T, Shinozuka T. Vaginal applicators (ovoids) for local control and alleviation of rectal complications of cervical cancers treated by brachytherapy. Tokai J Exp Clin Med 1999;24(1):2-7.

28. Potter $\mathrm{R}$, Kirisits $\mathrm{C}$, Lievens $\mathrm{Y}$. What kind of equipment do we need in gynecological brachytherapy? Clinical, technical and economical aspects for standard and new techniques. Radiother Oncol 2005;75 Suppl:S1.

29. Kirisits C, Potter R, Lang S, Dimopoulos J, Wachter-Gerstner N, Georg D. Dose and volume parameters for MRI-based treatment planning in intracavitary brachytherapy for cervical cancer. Int J Radiat Oncol Biol Phys 2005;62(3):901-11. 